Aus der Universitătsklinik für Hautkrankheiten in Bonn. (Direktor: Prof. Dr. E. Hoffmann.)

\title{
Weitere Mitteilungen über das Ehrlichsche Dioxydiamidoarsenobenzol (Salvarsan).*)
}

Von Dr. W. Heuck, Oberarzt der Klinik, und Dr. J. Jaffé, Assistenten der Klinik.

In der Sitzung der Niederrheinischen Gesellschaft für Natur- und Heilkunde am 18. Juli 1910 hat Hoffmann seine ersten Erfahrungen mit dem nunmehr Salvarsan benannten neuen Ehrlichschen Heilmittel (Dioxydiamidoarsenobenzol)

1) Noch ein anderer therapeutischer Eingriff ließe sich vernünftiger. weise hier versuchen, leider aber stehen der hiesigen Klinik die Mittel zu dessen Ausführung nicht zur Verfügung. Meine Turiner Klinik war mit einer pneumatischen Kammer für komprimierte Luft bis zu einer Atmosphäre versehen, und ich habe diese in manchen Fällen von Pneumothorax auch benutzt. Ließe man die Kranke einige Stunden lang - oder auch im Notfalle permanent - in der pneumatischen Kammer (wie in einem kontinuierlichen Bade) verweilen, so würde sie darin einen ihrer expiratorischen Insuffizienz genau angemessenen Ersatz finden (ihre Lungenventilation würde hierbei verdoppelt sein). Dies würde gestatten, die Vernarbung der rechten Lunge abzuwarten.

*) Nach einem in der Niederrheinischen Gesellschaft für Naturund Heilkunde, Sitzung vom 12. Dezember 1910, gehaltenen Vortrag. 
mitgeteilt und bald darauf in der "Medizinischen Klinik“"1) ausführlicher dargestellt. Es wurde von ihm die außerordentliche Wirkung des Salvarsans auf die verschiedenen Krankheitsformen der Syphilis an der Hand charakteristischer Krankheitsfälle anerkannt und speziell die Einwirkung auf dic in den frischen Krankheitsprodukten vorhandenen Spirochäten hervorgehoben. Ferner wurde der Werdegang der Arsentherapie der Syphilis und die Bedeutung der Spirochaeta pallida für die moderne Diagnostik und die Bekämpfung dieser Krankheit beleuchtet. Gleichzeitig wurden auch Tierexperimente angeführt, welche feststellen sollten, ob die im mikroskopischen Präparat kurze Zeit nach der Injektion von Salvarsan nicht mehr auffindbaren Spirochäten auch durch die Methode der Ueberimpfung sich nicht mehr nachweisen lassen.

Inzwischen haben wir weitere Erfahrungen an etwa 200 Kranken gewonnen und möchten neben den Ergebnissen der klinischen Beobachtung auch insbesondere über die Frage der Neberıwirkungen kurz berichten. Was zunächst die Dosierung bei den von uns mit Salvarsan behandelten Patienten betrifft, wurde, abgesehen von den ersten Fällen, die wir vorsichtiger mit $0,3 \mathrm{~g}$ behandelten, im allgemeinen $0,6-0,7 \mathrm{~g}$ bei Männern und $0,45-0,6 \mathrm{~g}$ bei Frauen angewandt; bei Kindern gaben wir etwa $8 \mathrm{mg}$ pro $\mathrm{kg}$ Körpergewicht. In erster Linie berïcksichtigten wir jedoch stets neben der Art der Erkrankung den Gesamtzustand des jeweils zu Behandelnden, ohne dabei die Dosis genau nach dem Körpergewicht zu berechnen, wie das in den Veröffentlichungen einiger Autoren, z. B. von Zieler, erwähnt wird. Aelteren, gebrechlichen Leuten gaben wir fast stets nur $0,3 \mathrm{~g}$, ebenso gingen wir bei Patienten mit starker Arteriosklerose, mit Aneurysmen, dann bei solchen mit metasyphilitischen Erscheinungen meist nicht über diese Dosis hinaus. Bei guter Verträglichkeit dürfte hier unserer Ansicht nach eine mehrmalige Injektion kleiner Mengen einer einzigen größeren vorzuziehen sein.

Die Zubereitung der Injektionsflüssigkeit erfolgte stets unmittelbar vor der Einspritzung, einmal zur Wahrung größtmöglicher Asepsis, dann aber, weil wiederholt beobachtet wurde, daß nach kurzem Stehen der Flüssigkeit eine spontane Ausfällung der vorher klaren oder fast klaren Lösung eintrat.

Vor de: Injektion wurde stets eine genaue Untersuchung der inneren Organe und des Augenhintergrundes vorgenommen.

Der Erörterung der von uns geübten Technik bei der Anwendung des Salvaisans sei vorausgeschickt, daß wir anfangs schwach saure Lösungen des Präparates benutzten. Wir gingen dann für kurze Zeit zum Gebrauch der älteren Wechselmannschen Methode über, wobei wir die neutrale Emulsion sowohl intramuskulär wie subkutan injizierten. Die große Mehrlieit der Patienten behandelten wir aber nach dem Altschen Verfahren, das in der Herstellung einer leicht alkalischen Lösung besteht. Nur kurz erwähnt seien die Injektionen von Aufschwemmungen des Salvarsans in Olivenöl (Volk), Paraffinum liquidum (Kromayer) oder Sesamöl im Verhältnis von 1 : 10, da wir über die Wirksamkeit dieser Methoden, die vor allem in der Einfachheit der Zubereitung iłıre Vorzüge haben dürften, keine eigene Erfahrung haben. In letzter Zeit sind wir auch zu intravenösen Injektionen übergegangen, deren Vorzüge ja auf der Hand liegen und von Schreiber und Weintraud immer wieder betont werden. Bezüglich der Technik dieser Infusionen verweise ich auf die Arbeit Géronnes.2) Abgesehen von vorübergehenden Störungen von seiten des Magen-Darmtraktus Jebelkeit, Erbrechen, Durchfälle - etwa drei bis vier Stunden nach der intravenösen Einverleibung und leichten Fiebersteigerungen, die aber nur bei wenigen Kranken auftraten, haben wir bisher keine unangenehmen Nebenerscheinungen auch keine Thrombosierung der zur Injektion gewählten Vene gesehen. ${ }^{3}$ ) Ueber die Resultate dieser Methode möchten wir

1) 1910 , No. 33 .

2) A. Géronne, Berliner klinische Wochenschrift 1910, No. 49

3) Anmerkung bei der Korrektur. Unter etwa 50 intravenösen Injektion`n erlebten wir einmal einen Kollaps mit starkem Erbrechen $(0,4$ intravenös nach 4 Wochen vorher gemachter intraglut $₫$ ealer Einspritzung von 0,5$)$, ferner ein erst am 6. Tage auftretendes universelles, morbillenähnliches, von hohem Fieber begleitetes Exanthem und eine $3 \mathrm{~cm}$ lange Thro in bose der V. cubitalis an der Injektionsstelle. jedoch heute bei der Kürze der Beobachtungszeit noch nicht berichten.

Zur Herstellung der von uns vorwiegend benutzten Lösung nach Alt bedienen wir uns eines graduierten, mit Glasstopfen versehenen Meßzylinders von $50 \mathrm{ccm}$, in welchen zunächst $8 \mathrm{ccm}$ heißes, destilliertes Wasser und dann das Präparat gebracht und mit Hilfe von Glasperlen durch kräftiges Schütteln aufgelöst wird. Alsdann wird auf $0,1 \mathrm{~g}$ des Präparates die Dosis von 0,5 Normalnatronlauge (4\% Natronlauge) zugesetzt, welche jedoch nicht immer ausreicht, um klare Lösung zu erzielen. Auf Grund der Erfahrung, daß ein zu starker Alkaleszenzgrad die Schmerzhaftigkeit der Injektion erhöht und auch die lokalen Entzündungserscheinungen steigert, sind wir bald dazu ïbergegangen, nach Zusatz von knapp $0,5 \mathrm{ccm}$ Normalnatronlauge auf $0,1 \mathrm{~g}$ des Präparates weiterhin nur tropfenweise Normalnatronlauge nicht bis zur klaren Lösung zuzusetzen, sondern nur soviel, bis die Wand des Meßzylinders beim Schütteln frei von ungelösten Partikeln bleibt, was mit einer deutlich erkennbaren Aenderung des Farbentons verbunden ist; so erhält man eine leicht getrübte Lösung, die gut einspritzbar ist und weniger Reizerscheinungen macht.

Eine Differenz in der Wirkung des Präparates je nach der Methode der Injektionstechnik konnten wir insofern konstatieren, als die subkutane Injektion der Emulsion nach W eclis el mann auf die klinischen Erscheinungen der Lues und die Spirochäten langsamer einwirkte, was freilich von Wechsel mann bestritten wird. - Bezüglich der Schmerzhaftigkeit und der lokalen Entzündungserscheinungen wurden die sauren Lösungen unserer Erfahrung nach am unangenehmsten empfunden; auch die später folgenden Reizerscheinungen in der Umgebung der Injektionsstelle waren entschieden heftiger und langdauernder als bei Anwendung der übrigen Methoden. Die viel gerühmte Schmerzlosigkeit der Wechselmannschen Methode können wir nach unseren Erfahrungen nicht uneingeschränlst bestätigen; in einigen Fällen war auch die subkutane Injektion im Gegenteil recht schmerzhaft, es bildeten sich sehr langdauernde, druckempfindliche Infiltrate, und in zwei Füllen kam es zu Nekrosen, einmal erst drei Monate nach der Injelstion zu einer obenflächlichen Nekrose von etwa Kirschgröße, ein anderes Mal fünf Wochen nach der Injektion zu einer solchen von Zweimarkstückgröße. In einem Falle, wo Fluktuation den Verdacht auf Abszeßbildung erregte, zeigten sich am zehnten Tage nach der subkutanen Injektion lediglich Reste der injizierten Masse in einer serösen Flüssigkeit, in einem zweiten ließ sich noch nach etwa fünf Wochen As-haltige Flüssigkeit nachweisen. Bei intramuskulärer Einverleibung dieser neutralen Emulsion wurden derartige langwierige und erweichende Infiltrate nicht beobachtet. Bei der am häufigsten von uns angewandten intramuskulären Injektion schwach alkalischer Lösung nach Alt waren die lokalen Erscheinungen äuBerst wechselnd und auch die subjektiven Beschwerden individuell sehr verschieden. Die Injektion selbst verursachte teilweise geringe, teilweise aber auch heftige Schmerzen, in seltenen Fällen blieben alle Beschwerden aus. Ein Patient konnte am Morgen nach der Injektion einen vielstündigen Spaziergang ohne jegliche Beschwerden und nachteilige Folgen unternehmen.

Jedoch haben die meisten Patienten unmittelbar nach der Altschen Injektion die unangenelıme Empfindung eines dumpfen Druckes oder gleich intensiven Schmerzes, der nach zwei bis drei Stunden gewöhnlich seinen Höhepunkt erreicht und etwa acht bis zehn Stunden anzuhalten pflegt. Eine Hauptklage bildet dann durchweg die Störung des Schlafes in den ersten Nächten. Nun folgt gewöhnlich eine Periode der Abnalıme der Beschwerden, bis nach etwa zwei bis drei Tagen, besonders wenn die für drei bis vier Tage angeordnete Bettruhe nicht eingehalten wird, von neuem Schmerzempfindung auftritt, die durch die dann meist einsetzenden lokalen Erscheinungen Hautrötung, mehr oder weniger hochgradige Schwellung der Hinterbacke und Infiltration - bedingt ist. In seltenen Fällen kommt es, offenbar unter dem Druck des Infiltrates, zu mehr oder weniger ausgesprochenen neuralgischen Schmerzen, besonders an der Außenseite des Knies und der Wade. Lähmungen oder Paresen sind uns nie vorgekommen. Seitdem 
wir die Gesamtmenge der Lösungsflüssigkeit, die wir anfangs auf etwa $15 \mathrm{ccm}$ bemaßen, wie oben angegeben, auf knapp $8-10 \mathrm{ccm}$ reduziert haben, sind die lokalen Reizerscheinungen und Beschwerden entschjeden geringer geworden, besonders wenn eine drei- bis viertägige Bettruhe zur Vermeidung eines stärkeren Infiltrats eingehalten wurde. Treten trotzdem stärkere Beschwerden auf, so läßt sich durch lokale feuchtwarme Umschläge mit essigsaurer Tonerdelösung, durch heiße Sitzbäder und leichte Massage, ev. unterstützt durch interne Beruhigungsmittel, sofort eine Milderung und meist in einigen Tagen guter Rückgang der akuten Entzündungserscheinungen herbeiführen.

Temperatursteigerungen, begleitet von Erhöhung der Pulsfrequenz, waren in einem gewissen Prozentsatz bei allen drei Methoden zu verzeichnen. Die Temperatur stieg bei einigen wenigen Patienten über $\mathbf{3 9 , 5}$ bei einer Pulsfrequenz von 120 bis 140 , meist jedoch nicht wesentlich über $38^{\circ}$. Ein bestimmter Fiebertypus ließ sich nicht konstatieren. Gewöhnlich stieg die Temperatur bereits einige Stunden nach der Injektion an, um am anderen Morgen zur Norm abzufallen, in anderen Fällen behielt die Temperaturerhöhung zwei bis drei Tage hindurch einen remittierenden oder intermittierenden Charakter und erreichte gleichzeitig mit den lokalen Reizerscheinungen ihren Höhepunkt, um dann kritisch oder lytisch abzufallen. Bei einigen Patienten setzte ein Fieber des oben geschilderten unbestimmten Charakters erst nach zwei bis drei Tagen ein. Bei den mit saurer Lösung behandelten Patienten waren in etwa einem Drittel der Fälle solche Fiebersteigerungen zu beobachten, ohne daß jedoch der Allgemeinzustand wesentlich beeinträchtigt gewesen wäre. Eine Ausnahme bildet ein von Hoff mann in seiner Veröffentlichung ${ }^{1}$ ) erwähnter Fall einer zentralen Pneu monie, welche wahrscheinlich durch Embolie infolge einer durch die saure Lösung am Orte der Injektion verursachten Thrombose hervorgerufen wurde. Einen ähnlichen Fall erwähnt auch G éronne aus der Abteilung Weintrauds, ${ }^{2}$ ); hier kam es 24 Stunden nach der intramuskulären Injektion zu einer von hohem Fieber begleiteten Pleuritis, die mutmaßlich durch Embolie veranlaßt war. Bei Befolgung der Altschen Methode war das Fieber unbedeutend und fehlte in einem großen Teil der Fälle vollkommen. Die Injektionen müssen selbstverständlich unter genauester Befolgung aller Regeln der Asepsis und recht langsam vorgenommen und so tief gesetzt werden, daß das Präparat in die Substanz des Glutaeus maximus gelangt. Ebenso wie bei den Injektionen von Quecksilberpräparaten ist auch hier der Lesser sche Handgriff zur Vermeidung der Injektion der Lösung in die Blutbahn wohl zu beachten. Von Wichtigkeit ist ferner die Vermeidung des Eindringens von Flüssigkeit in den Stichkanal, was wir dadurch zu erreichen suchen, daß wir zunächst die Kanüle mit physiologischer Kochsalzlösung gefüllt einführen und beim Herausziehen nach der Injektion die umgebenden Muskeln fest um die Kanüle herumpressen. Bei peinlicher Befolgung dieser Maßregeln können unserer Erfahrung nach Nekrosen der Haut vermieden werden, die bei subkutaner Injektion verhältnismäßig häufig auftreten. In der Glutäalmuskulatur kommt es aber infolge der ätzenden Wirkung des Präparats stets zu tiefen, unter der intakten Hautdecke sich entwickelnden Nekrosen von mehr oder weniger großem Umfang, wie sie von Orth und anderen beschrieben worden sind. Auch wir konnten eine solche auffallend große Nekrose der Muskulatur bei der Sektion einer Patientin beobachten, die $31 / 2$ Wochen nach der intramuskulären Injektion alkalischer Salvarsanlösung infolge schwerer viszeraler Lues starb. Obwohl die Patientin keine sehr erheblichen Beschwerden an der Injektionsstelle gehabt hatte und auch die lokalen Entzündungserscheinungen nicht besonders stark gewesen waren, fand sich bei der Autopsie eine in der Faserrichtung des Glutaeus maximus verlaufende ausgedehnte Nekrose von etwa $10 \mathrm{~cm}$ Länge und $3 \mathrm{~cm}$ Durchmesser. Diese durch die Aetzwirkung des Salvarsans bedingten Muskelnekrosen erklären uns die fast stets vorhandenen heftigen Beschwerden und die hochgradige reaktive Entzündung; sie bilden sich langsam zurück und lassen sich auch klinisch noch wochenlang als derber Strang nachweisen.

1) ef. loc. cit. - - 3) of. loc. (it.
Zu einer Nekrose der Haut geben sie aber bei richtiger Technik unserer Erfahrung nach niemals Anlaß; eine solche kommt vielmehr nur dann zustande, wenn die ätzende Masse ins subkutane oder gar kutane Gewebe deponiert und durch Gefäßverschluß der Haut ihre Ernährung abgeschnitten wird. Auch aus diesem Grunde haben wir die subkutane Injektion, die häufig Nekrose der Haut oder langdauernde derbe Infiltrate im Gefolge hat, völlig aufgegeben.

Starke Pulsbeschleunigung und Herzgerausche, die von Hoffmann zuerst beschrieben wurden, dann aber auch von anderer Seite konstatiert werden konnten, traten bei einer kleinen Anzahl von Patienten in unmittelbarem Anschlusse an die Injektion auf. Die Herzgeräusche waren auf die Gegend der Mitralis beschränkt, bei einigen Patienten aber auch über allen Ostien zu hören; sie erwiesen sich stcts als vorübergehend und verschwanden, ohne dauernde Schädigung zu hinterlassen. Bei einer mit Mitralinsuffizienz behafteten Patientin hatte die intramuskuläre Injektion keinen schädigenden Einfluß auf die Herzaffektion und ebensowenig eine intravenöse Injektion von 0,3 bei einem Patienten mit ausgesprochener Aorteninsuffizienz. Eine wohl embolische zentrale Pneumonie und Pleuritis, die hier zur Beobachtung kam, wurde bereits oben erwähnt. Nachteilige Beeinflussung der Gravidität, wie sie von anderer Seite schon berichtet worden ist und als Kontraindikation gegen die Anwendung des Mittels angeführt wurde, konnte bei uns - in zwölf Fällen der verschicdensten Stadien der Schwangerschaft - nicht beobachtet werden. Sehnervenerkrankungen, wie sie nach Atoxyl- und Arsazetingebrauch sich einste. ten, kamen uns nicht zu Gesicht ${ }^{1}$ ); im Gegenteil kam eine spezifische Chorioiditis nach der Injektion zur Abheilung. Eine beginnende Optikusatrophie bei einer Patientin mit tertiär-luetischen Hauterscheinungen erfuhr keine Verschlimmerung. Ebenso sahen wir keine Schädigungen des Gehörs, keine Tenesmen von Blase und Mastdarm, nur bei einem Patienten trat vorübergehend Harndrang auf. Auftreten von Albumen im Urin konnte nie festgestellt werden; im Gegenteil verschwand in einem Falle nach der Injektion von $0,7 \mathrm{~g}$ in einigen Tagen eine mit syphilitischen Allgemeinerscheinungen wieder aufgetretene ältere Albuminurie. Einmal wurde bei einem Patienten Prof. Hoff manns nach der Injektion eine eintägige Glykosurie beobachtet. Bei einem anderen, bei dem kurz zuvor nach stärkerem Sektgenuß Glykosurie aufgetreten war, brachte die Injektion von Salvarsan keine Schädigung, die Zuckerausscheidung verschwand kurz nach der Injektion. Die unerwünschten Nebenwirkungen bei den Injektionen dürften sich also nach unseren Erfahrungen in der Hauptsache auf lokale Beschwerden beschränken, dauernde Störung von wichtigen Organen und Organfunktionen aber im allgemeinen nicht zu befürchten sein.

Unter den Nebenerscheinungen sind hier noch Erytheme zu erwähnen, die wir in einer kleinen Anzahl der Fälle mehrere Stunden nach der Injektion oder am nächsten Tage auftreten sahen und die sich deutlich von einer lokalen Reaktion unterschieden. Sie hatten meist urtikariellen Charakter, zum Teil bestanden sie jedoch aus papulösen Effloreszenzen, die Extremitäten und Rumpf bedeckten. Ein besonders bemerkenswertes Exanthem sahen wir bei einem mit 0,6 $\mathrm{g}$ nach der Wechselmannschen Methode intraglutäal behandelten $\mathrm{Pa}-$ tienten acht Tage nach der Injektion auftreten. Unter mehrtägigen anhaltenden Fieberbewegungen von 39,6-40,2 , Herzgeräuschen über allen Ostien und starken Allgemeinstörungen setzte eine schwere Angina mit dickem fibrinösen Belag und gleichzeitig ein papulöses Erythem ein. Dieses hatte größtenteils den Charakter eines Erythema exsudativum (papulatum), war auf Rumpf, Extremitäten und Gesicht diffus verteilt und besonders intensiv in der Nachbarschaft der Injektionsstelle, an der sich gleichzeitig ein hartes Infiltrat bildete. Es gehört dieser Fall zu den Beobachtungen von sog. ,Spätreaktionen“,

1) Hier soi erwähnt, daß Prof. Hoffmann in seiner Privatpraxis inzwischen eine einseitige Neuritis optica beobachtet hat, die als echtes Luesrezidiv gut zwei Monate nach der Injektion von 0,6 alkalischer lösung intraglutäal auftrat und durch $\mathrm{Hg}$ und Jodkali bald gebessert wurde. Störungen von seiten des Acusticus oder anderer Nerven wurden nicht beobachtet. 
wie solche, in ihrem klinischen Bild fast übereinstimmend, von Wechselmann, Lesser, Goldbach u. a. beschrieben und in ihrer Bedeutung hervorgehoben worden sind; es handelt sich hier höchstwahrscheinlich um toxische As-Exantheme bei besteherder Ueberempfindlichkeit. ${ }^{1}$ ) Lokale Reaktion ( $\mathrm{Ja}$ risch-Herxheimer) konnten wir in vereinzelten Fällen bei Sklerosen, in der weitaus überwiegenden Mehrzahl bei den sekundär luetischen Exanthemen beobachten. Bemerkenswert ist noch die starke lokale Reaktion bei tertiärer Lues, bei einer Patientin mit tubero-scrpiginösem lupoiden Syphilid des Gesichtes, die von Hoffmann in seiner oben zitierten Arbeit bereits erwähnt worden ist. Als Reaktion dürfte wohl auch die vorübergehende Steigerung bestehender Kephalalgie zu betrachten sein.

Derartige Reaktionen auch bei Späterscheinungen der Lues lassen vermuten, daß solche auch bei syphilitischen Affektionen innerer Organe (Herz, Kehlkopf) und des Zentralnervensystems eintreten können, und mahnen hier zur Vorsicht in der Dosierung. Durch einen derartigen Reaktionsvorgang ist, wie man annimmt, ein Todesfall nach Salvarsaninjektion in der hiesigen Nervenklinik zu erklären, bei dem sich bei genauer mikroskopischer Untersuchung ein syphilitisches Infiltrat um den N. phrenicus an dessen Austrittsstelle aus dem cberen Halsmark fand und Atmungsstil stand bewirkte.

Bei der Schilderung der klinischen Wirkung des Salvarsans sei zunächst seine Einwirkung auf Primäraffekte angeführt. Berücksichtigt sind im folgenden lediglich Fälle mit mindestens vierwöchiger Beobachtungszeit.

Bei den Fällen reiner primärer Lues war die Wirkung des Mittels wechselnd, je nach der Beschaffenheit des Primäraffektes. Oberflächliche erodierte Sklerosen epidermisierten sich stets in zwei bis drei Tagen und waren alle in fünf bis 14 Tagen vollkommen abgeheilt, d. h. jede spezifische Härte verschwunden. Hierunter befanden sich auch mehrere Fälle mit multiplen, einer sogar mit 17 Primäraffekten. Es waren im ganzen acht Fälle, von denen drei etwa acht Tage nach der Injektion noch einer $\mathrm{Hg}$-Kur unterzogen wurden. Die stark indurierten, zum Teil knorpelharten, zum Teil ulzerierten Sklerosen - im ganzen neun - zeigten sich hartnäckiger und benötigten bis $\mathbf{z u}$ ihrer Abheilung mindestens vier Wochen. Nur drei von diesen wurden mit Salvarsan allein behandelt, drei andere gleichzeitig auch einer Hg-Kur unterzogen und heilten in vier bzw. sechs Wochen, drei weitere Fälle wurden durch Exzision beseitigt. Bei den kombiniert Behandelten trat zweifellos eine bessere Rückbildung sowohl des sklerosierten Gewebes als auch der regionären Drüsen ein. Alle diese 17 Fälle von reinen Sklerosen blieben in der bisherigen Beobachtungszeit teilweise sechs Monate hindurch frei von lokalen Rezidiven und Allgemeinerscheinungen.2)

Ferner wurden sieben Fälle von Primäraffekten mit bereits bestehenden Sekundärerscheinungen behandelt, vier mit Salvarsan, drei zugleich mit $\mathrm{Hg}$; auch sie heilten in zwei bis zwölf Wochen, je nach der Stärke des Infiltrats. Während sich die mcisten Sklerosen auffallend schnell überhäuten und die oberflächlichen auch rasch zurückbilden, gehen stark infiltrierte langsam zurück; letzteres wird ja auch bei der $\mathrm{Hg}$-Behandlung gewöhnlich beobachtet. In all diesen, auch den gleichzeitig mit Hg-Kur behandelten Fällen fand eine lokale Behandlung nicht statt.

Gute Rückbildung der Drüsen erfolgte bei den primär luetischen Patienten nur in wenigen Fällen. In der Mehrzahl wurden sowohl die allgemeinen wie die regionären Drüsenschwellungen nicht sehr schnell und deutlich beeinflußt.

Die verschiedenen Erscheinungen sekundärer Lues zeigten sich ungleich in ihrer Beeinflußbarkeit durch das Salvarsan. Bei der Behandlung mit Salvarsan allein verschwanden die makulösen Exantheme fast durchweg in drei bis sieben Tagen, nur vereinzelte benötigten 10 bis 14 Tage bis $\mathrm{zu}$ ihrer Abheilung. Papulöse Exantheme gingen langsamer zu-

1) Einmal wurde ferner ein abortiver Zoster beobachtet.

2) Anmerkung bei der Korrektur. Inzwischen ist bei einem mit 0.5 intraxlutaeal gespritzten Patienten. dessen Sklerosen zugleich exzidiert wurden, nach $51 / 2$ Wochen ein lokales Rezidiv (Sp. p. +, W. R. -) aufgetreten. rück, nicht vor einer Woche; drei waren erst nach vier bis sechs Wochen geheilt. Von 14 Fällen ausschließlich mit Salvarsan behandelter Genitalpapeln heilten neun in 3 bis 14 Tagen ab. Von den übrigen sechs verschwanden drei in vier Wochen, zwei waren nach vier Wochen noch ungeheilt und heilten erst sieben Tage nach einer zweiten Injektion, während ein mit 0,6 behandelter Fall mit Analpapeln nach drei Monaten noch ungeheilt war und neuerdings einer zweiten Injektion unterzogen wurde, die bis jetzt, nach weiteren fünf Wochen, nur eine Besserung, keine Heilung brachte. Ausgezeichnet wurden sekundäre Schleimhaute sscheinungen beeinflußt. Anginen heilten längstens nach sieben, die meisten schon nach drei oder fünf Tagen, nur eine ulzeröse brauchte 14 Tage, und Lippenpapeln verschwanden nach durchschnittlich einer Woche. Ein Fall von Zungenpapeln blieb nach drei Wochen bis jetzt noch ungeheilt, die gleiche Dauer ein bis zwei Wochen - erforderte die Impetigo capitis, eine Irispapel heilte in vier Tagen ab.

Es war also bei den Sekundärerscheinungen die therapeutische Wirkung des Mittels sehr ungleichmäßig, überraschend schnell bei den meisten Schleimhauterscheinungen, langsamer besonders bei einer Anzahl von Genitalpapeln, namentlich den luxurierenden. Bei drei von diesen Fällen sahen wir uns, wie erwähnt, zu einer zweiten Injektion veranlaß3t, und nur bei zweien wurde hier nach einer Gesamtdosis von $1,2 \mathrm{~g}$ eine Abheilung erreicht, während ein dritter sich refraktär erwics. Andere Fälle sekundärer Lues wurden einer kombinierten Behandlung mit $\mathrm{Hg}$ unterzogen, 13 von diesen sind wenigstens vier Wochen beobachtet. Ein wesentlicher Unterschied in der Dauer der Abheilung gegenüber den mit Salvarsan allein behandelten Fällen ergab sich nicht. Ein Teil der Erscheinungen war bereits vor Einsetzen der Hg-Kur, die mehrfach erst nach acht bis 14 Tagen begonnen wurde, verschwunden; zwei besonders hartnäckige papulöse Syphilide brauchten trotz der kombinierten Kur sechs bis sieben Wochen zu ibrer Abheilung. Genitalpapeln heilten in einem Falle erst nach sechs Wochen, in zwei Fällen nach vier Wochen. Eine lokale Behandlung der Erscheinungen wurde auch hier in allen Fällen nicht vorgenommen.

Von den Fällen maligner Lues steht uns zur Beurteilung nur ein genügend lange beobachteter zur Verfügung. Der Patient war Träger eines ausgedehnten ulzerösen Syphilids der Haut und ulzerierter Plaques der Zunge; diese Erscheinungen heilten auf eine Injektion von 0,3 Salvarsan in vier Wochen ab, um jedoch bereits eine Woche später von neuem aufzutreten. Eine zweite Injektion von 0,6 führte in etwa 14 Tagen zur Abheilung.

Bei zwölf Fällen tertiärer Syphilis konnten wir fast stets eine schnelle Rückbildung der Haut-, Schleimhaut- und Periosterkrankungen beobachten, ebenso verschwanden auffallend schnell - mitunter bereits wenige Stunden nach der Injektion - hochgradige nächtliche Kopf- und Knochenschmerzen.

Ein über das ganze Gesicht ausgedehntes ulzero-serpiginöses lupoides Syphilid (von Hoffmann bereits beschrieben) heilte in 14 Tagen nach 0,3 Salvarsan vollkommen ab und ist bisher (nach 6 Monaten) nicht rezidiviert. Gummöse Ulcera von beträchtlicher Größe sahen wir in verhältnismäßig kurzer Zeit zur Verheilung kommen, solche von etwa Dreimarkstückgröße in etwa 14 Tagen, ein tiefes, fast faustgroßes phagedaenisches gummöses Ulcus (Phagedénisme tertiaire Fourniers) besserte sich mit überraschender Schnelligkeit und war nach $3 \frac{1}{2}$ Wochen fast geschlossen; vor vollkommener Heilung der Hauterscheinungen trat infolge schwerer viszeraler Lues mit Peritonitis und fast völligem Verschluß der V. cava inferior, die das Mittel nicht zu beheben vermochte, der Tod ein.

Ein großer gummöser Zerfall am Oberkieferzahnfleisch verheilte in fünf Wochen, ein tiefes Zungengumma sowie ulzerierte Gummata am Scrotum fast vollkommen in zwölf. Tagen; im letzten Falle trat allerdings bald ein Rezidiv ein. Periostitische Auftreibungen einer an Leberlues leidenden Patientin verschwanden in sieben Tagen; die Leber. vergrößerung ging auch deutlich zurück, doch ist hier noch weitere Beobachtung erforderlich. - Eine Uveïtis specifica besserte sich bereits acht Tage nach der Injektion erheblich und war geheilt, als Patientin sich nach zwei Monaten wieder vorstellte. Von zwei Patienten mit Ozaena syphilitica wurde bei einem mit Salvarsan allein lediglich eine recht gute Besserung, jedoch keine Heilung erzielt, die Erscheinungen 
heilten dann nachhel auf Jodkaligebrauch. Bei einem anderen Pa. tienten mit Ozäna und gummösen Ulcera des Naseninneren erfolgte fast völlige Heilung durch Salvarsan allein.

Ein ganz verzweifelter Fall von Lues congenita tarda ( 15 jähriger Knabe) mit völliger Zerstörung des Naseninnern bis zur Schädelbasis, Vortreibung eines Auges, drohender Meningitis und unerträglichem Kopfschmerz, der durch hohe Joddosen und Kalomelinjektionen nicht geheilt werden konnte, zeigte, wie Hoffmann bereits geschildert hat, schon nach 0,3 eine sehr schnelle, außerordentliche Besserung mit fast sofortigem Nachlaß der Schmerzen und des penetranten Gestanks. Als später die Borkenbildung wieder zunahm, brachte eine zweite Injektion von 0,6 wiederum einen noch anhaltenden, recht guten Erfolg in diesem vorher völlig aufgegebenen Falle.

Ein zwölfjähriges Mädchen nit Lues congenita tarda, das sehr hartnäckige, ausgedehnte gummöse Ulzerationen an einem Vorderarm und Unterschenkel hatte, wurde durch zwei intramuskuläre Injektionen von je 0,16 vollkommen geheilt und blieb es bis jetzt.

Wir haben außerdem cine Anzahl Patienten mit metasyphilitischen Erscheinungen, Tabes, beginnender Paralyse, mit Salvarsan behandelt. Bei diesen schweren organischen Veränderungen konnte übereinstimmend mit den Resultaten der meisten Beobachter ein sichtlicher Erfolg nicht konstatiert werden, wenn auch bei einzelnen Patienten vorübergehende Besserung subjektiver Beschwerden, einmal auch der trägen Pupi:enreaktion erzielt wurde.

Zusammenfassend können wir sagen, daß die klinische Wirkung des Salvarsans in allen Stadien der Syphilis, abgesehen von den metasyphilitischen Erkrankungen, recht gut genannt werden muß. Am hartnäckigsten erwiesen sich die Drüsenschwellungen, derbe Sklerosen und manche sekundäre papulöse Exantheme. Besonders hervorheben müssen wir die schnelle and vollständige Heilung der oberflächlichen Sklerosen, der sekundären Schleimhauterscheinungen, die hervorragend günstige Becinflussung der tertiären - auch tardiven kongenitalen - Haut- und Schleimhauterscheinungen, sowic die außerordentlich schnelle Beseitigung der starken subjektiven Beschwerden, wie z. B. der Knochenschmerzen auch in verzweifelten und sonst nicht becinflußbaren Fällen.

Völlige Versager, in denen jede nachweisbare Einwirkung fehlte, haben wir mit Ausnahme der metasyphilitischen Erscheinungen in der Klinik nicht gesehen, nur bei einem sokundär luetischen Patienten aus der Privatpraxis von Herrn Prof. Hoffmann, der anderwärts eine Injektion von 0,6 Salvarsan (noutrale Aufschwemmung) erhalten hatte, blieben die Erscheinungen - Angina specifica und Papeln an Anus und Penis - ganz unbeeinflußt and heilten erst durch eine $\mathrm{Hg}$ salizylkur.

Rezidive sind dagegen auch bei uns beobachtet, und zwar unter 77 mit Salvarsan behandelten und mindestens vier Wochen nach der Injektion weiter beobachteten Patienten mit luetischen Haut- oder Schleimhauterscheinungen sjeben. $\left.{ }^{1}\right)$

Eine Patientin mit Primäraffekt und einzelnen frischen Roseolen bekam drei Wochen nach der Injektion von $0,5 \mathrm{~g}$ (Alt) eine großfleckige, ausgedehnte Roseola von t $€$ ilweise zirzinärer und annulärer Anordnung, die sich auch anf Arme und Beine erstreckte und nach einer zweiten Injektion verschwand. Bei einer Patientin mit Angina specifica und starkem papulösen Syphilid traten etwa acht Wochen nach der Injektion von $0,3 \mathrm{~g}$ (saurer Lösung) und $\mathrm{Hg}$-Kur (Inunktionen) eine ulzeröse spezifische Angina sowie mäßig zahlreiche Rupiaeruptionen an beiden Armen, ${ }^{2}$ ) bei der anderen fünf Monate nach der subkutanen Injektion von $0,4 \mathrm{~g}$ sowie ebenfalls vollendeter $\mathrm{Hg}$-Kur eine Roseola und spezifische Angina auf. Außərdem kam ein Rezidiv bei dem oben erwähnten Fall von maligner Lues fünf Wochen nach Injektion von $0,3 \mathrm{~g}$ Salvarsan vor. Ferner rezidivierten ulzerierte Gummen der Zunge und des Scrotums etwa sechs Wochen nach der Injektion von $0,3 \mathrm{~g}$ in saurer Lösung und ein orbikuläres Syphilid am Penis bei einem Privatpatienten von Herrn Prof. Hoffmann, der anderwärts gespritzt war und sechs Wochen nach schneller Heilung an der gleichen Stelle dieselbe Eruption zeigte, die nach $20 \mathrm{~g}$ Jodkali völlig verschwand. Durch $\mathrm{Hg}$ und $\mathrm{Jk}$ wurde bald Besserung erzielt.

1) Anmerkung bei der Korrekt.ur: Inzwischen ist diese Zahl auf 15 gestiegen.

2) Die Wassermannsehe Reaktion war hier bei Auftreten des Rezidivs längere Zeit negativ bei positivem Spirochätenbefund auf den Tonsillen. Solch vorübergehende, ,negative Phase“ (Hoffmann) der Wassermannschen Reaktion nach einer Kur konnten wir wiederholt noch bei Einsetzen eines Rezidivs konstatieren.
Als Rezidiv möchten wir auch eine Neuritis optica auffassen, die bei einem Privatpatienten von Prof. Hoff mann sieben Monate nach der Infektion einsetzte, nachdem zweieinhalb Monate vorher eine Injektion von 0,6 Salvarsan wegen eines Rezidivs (Roseola, Angina specifica, Zungenplaques, Phlebitis der V. saphena parva) gemacht worden war.

Kurz vor der Injektion hatte eine genaue Untersuchung normalen Befund auf beiden Augen ergeben; etwa 21/2 Monate danach klagte Patient über Sehstörungen, Nebel und Druckschmerz auf dem linken Auge. Die Untersuchung in der Augenklinik ergab nun rechts normalen Befund, links war der äußere Augenbefund normal, das ophthalmoskopische Bild zeigte jedoch hier eine vergrößerte, grau verfärbte, nicht prominente Papille mit völlig verwaschenen Grenzen. Die Arterien waren eng, die Venen ziemlich stark gefüllt. Das Gesichtsfeld war für weiß normal, für grün und rot leir ht eingeengt. Die Sehschärfe betrug links die Hälfte der normalen. Durch $\mathrm{Hg}$ und $\mathrm{Jk}$ wurde bald Besserung erzielt.

Ueber Nervenerkrankungen und insbesondere Hirnnervencrkrankungen - Opticus, Acusticus - nach Salvarsangebrauch ist ja in letzter Zeit wiederholt von Buschke, Finger, Wechselmann, Kowalewski, Blaschko u. a. berichtet worden. Einige Autoren führen sie auf eine dem Salvarsan eigene neurotrope giftige Wirkung zurück. Ehrlich, der in einem Vortrag in Frankfurt kürzlich selbst das Wort zu dieser momentan so sehr im Vordergrund des Interesses stehenden und wichtigen Frage ergriffen hat, hält diese vermeintlichen Nervenschädigungen für syphilitische Frührezidive. In der Tat befanden sich bisher die von derartigen Affektionen befallenen Patienten alle im Frühstadium, und auch ohne Behandlung und nach Hg-Kuren kommen derartige Nervenrezidive bei rezenter Syphilis vor. Jedenfalls möchten wir eine direkte toxische Wirkung des Salvarsans zunächst nicht annehmen. Bisher haben wir solbst keine nachweisbare Schädigung des optischen Apparates - auch bei beginnender Optikusatrophie - gesehen, und auch unter 1400 mit Salvarsan behandelten Fällen auf der Abteilung Wechselmanns kamen bisher keine Sehnervenerkrankungen zur Beobachtung. Sollten sich solche Fälle häufen, müßte man allerdings annehmen, daß das Salvarsan das Auftreten derartiger Rezidive begünstigt. Jedenfalls verdienen diese Fälle ganz besondere Aufmerksamkeit, damit die Bedingungen, unter denen sie auftreten bzw. vermieden werden können, bald klargestellt werden. Ehrlich glaubt, daß sie vor allem beim Gebrauch der subkutanen Injektion neutraler Aufschwemmung eintreten, und rät daher zur intravenösen Behandlung:

Die Becinflussung der Syphilisspirochäten in den offenen und infektiösen Produkten der rezenten Syphilis nach einer Injektion mit Salvarsan ist äuBerst überraschend. Sie wurde in allen solchen Fällen, wo eine Untersuchung möglich war, mit der Dunkelfeldmethode vor und nach der Injektion ausgeführt und täglich, teilweise in Abständen von Stunden, aufs genaueste verfolgt. An der Oberfläche der offenen Primäraffekte nehmen die Spirochactac pallidae bereits nach sechs bis acht Stunden an Zahl sehr bodeutend ab und verschwinden gewöhnlich nach ein- bis zweimal 24 Stunden, ebenso in sekundären Schleimhauteffloreszenzen und Genital- und Analpapeln; in drei Fällen verschwanden sie in letzteren erst am vierten bis fünften Tage. Wir haben uns aber nicht damit begnügt, diese Wirkung auf die Syphilisspirochäten im oberflächlichen Sekret zu konstatieren, vielmehr auch in der Tiefe des Gewebes nach Exzision von Primäraffekten und Papeln nach Spirochäten gefahndet (Gewebssaftmethode Hoffmanns) und sie hier nicht nur im frischen, sondern auch im Silberpräparat darzustellen versucht. Dabei haben wir fast stets die Spirochäten schon nach ein bis drei Tagen nicht mehr nachzuweisen vermocht, nur in ganz vereinzelten Fällen fanden wir sie später, so in einer großen Sklerose noch am achten Tage nach Wecliselmannscher Injektion, in Analpapeln am fünften bis sechsten Tage.

Nun entstand die praktisch wichtige Frage, ob die Infektiosität auch wirklich erloschen sei. Zu diesem Zweck haben wir auf Veranlassung von Prof. Hoff mann 24 Stunden bis vier Tage nach der Injektion Stückchen von exzidierten Sklerosen und Papeln Kaninchen in beide vorderen Augenkammorn und zum Teil auch in die Hoden und Hodenhaut 
eingeimpft. Diese Versuche sind an einer größeren Zahl von Kaninchen ausgeführt worden, von denen fünf nunmehr drei bis vier Monate in Beobachtung sind und keinerlei Krankheitszeichen aufweisen. Allerdings entsteht die Frage, ob Kaninchen, welche nach $\mathrm{H}$ off manns Erfahrungen bei Impfung in beide Augenkammern mit Passagerirus fast regelmäßig erkranken, für solche Experimente mit menschlichem Virus genügend empfindlich sind. Wir haben deshalb auch Affen geimpft und bei einem Tier, das mit 24 Stunden nach der Injektion exzidierten Papeln inokuliert worden war, Primäraffekte mit reichlichen, gut beweglichen Spirochaetae pallidae auftreten sehen; in mehreren anderen Versuchen wurde bisher ein positives Ergebnis nicht erzielt.

Nach diesen noch nicht abgeschlossenen Experimenten, die, wie wir selbst wissen, bindende Schlüsse noch nicht erlauben, scheint die Virulenz und Ueberimpfbarkeit der Spirochaeta pallida durch die Injektion des Salvarsans nach 48 Stunden bereits erheblich herabgesetzt zu sein.

Einen bemerkenswerten EinfluB des Salvarsans auf andere Spirochäten konnten wir nicht konstatieren. Das Verhalten der Mundspirochäten bildet nach unseren Beobachtungen jedenfalls kein Kriterium für die Wirkung auf die Syphilisspirochäten, wie Gerber anfangs angenommen hat; wir konnten Mundspirochäten vor wie nach einer Injektion feststellen, ebenso kounten wir in mehreren Fällen viele Tage nach der Injektion noch Spirochaeta refringens in dem Oberflächensekret und in den Gewebsabstrichen von Papeln und Papillomen in reichlicher Menge nachweisen.

Die Beeinflussung der Wassermannschen Reaktion durch Salvarsan war in den einzelnen Stadien sehr verschieden. ${ }^{1}$ ) Bei den 17 Fällen reiner pr i märer Lues blieb sie in sechs Fällen, wo sie vor der Injektion negativ befunden worden wa', bisher stets negativ. Hiervon sind zwei vier Wochen, drei drei Monate, einer fünf Monate beobachtet; in einem anderen Falle wurde die vorher negative Reaktion bald nach der Injektion schwach positiv, um nach drei Wochen wieder negativ zu werden und bisher bei einer mehrmonat ichen Beobachtung negativ zu bleiben. In den anderen zehn Fällen wurde die anfangs mehr oder weniger ausgesprochene positive Wassermannsche Reaktion stets negativ. In zweien dieser Fälle, wo sie anfangs nur schwach positiv war, wurde sie bereits nach zwei bis drei Wochen negativ, in den anderen Fällen erst nach drei bis sechs Wochen. Auch hier sahen wir mitunter vorübergehend eine Verstärkung der positiven Reaktion, der dann die negative Phase folgte.

Ein deutlicher Unter'schied in der Beeinflussung der Wasserunannschen Reaktion bei reiner Behandlung mit Salvarsan und bei mit $\mathrm{Hg}$ kombinierter Behandlung ließ sich bei den Fällen ron primärer Lues nicht feststellen; immerhin wurden einige kombiniert behandelte Fälle anscheinend etwas früher negativ. Bei den Fällen von sekundärer Lues mögen hier nur solche angeführt werden, die mindestens zwei Monate in Beobachtung standen. Es ergibt sich dann, daß im ganzen von 26 Fällen zwar 18 negativ wurden, aber nur 13 negativ blieben, bei fünf trat wieder ein Umschwung der negativ gewordenen zu stark positiver Reaktion auf; in vier dieser Fälle begleitet von klinischen Rezidiverscheinungen.

Bei den 18 negativ gewordenen Fällen erfolgte die Umstimmung frühestens nach vier Wochen. Von den allein mit Salvarsan behande ten Fällen wurden nur $53 \%$, von den zug eich mit Hg-Kur behandelten aber $30 \%$ negativ, ein Resultat, das für die bessere Wirkung der kombinierten Behandlung spricht. Bei dem einen Patienten mit maligner Lues ging die Wassermannsche Reaktion parallel den Erscheinungen, indem sie nach Abheilung der ersten Erscheinungen negativ und bei Eintritt des Rezidivs wieder positiv wurde. Bei den tertiär syphilitischen und bei den kongenital syphilitischen Patienten (Lues congenita tarda), die alle lediglich mit Salvarsan behandelt wurden, konnte trotz der güinstigen klinischen Wirkung des Mittels keine Aenderung der stets stark positiven serologischen Reaktion, abgesehen von einem kurz vorher auch mit $\mathrm{Hg}-\mathrm{Kur}$ behandelten Falle, erzielt werden.

1) Diese Untersuchungen wurden von Herrn Dr. Oelgart in der Klinik ausgeführt.
Eine Uebersicht über dieses Verhalten der Wassermannschen Reaktion gibt nebenstehende Kurve:

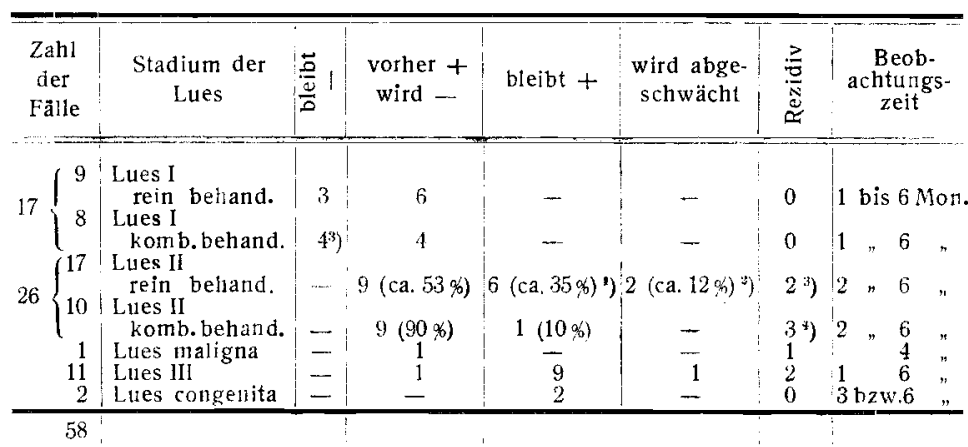

Außer den hier berichteten Beobachtungen über die Wirkung des Salvarsans auf die Lues in ihren verschiedenen Stadien seien dann noch Versuche einer Beeinflussung anderer Erkrankungen angeführt. Bemerkenswert ist zunächst die Einwirkung auf Warzen, und zwar nicht nur auf Verrucae planae juveniles, die durch Arsen ja öfters beeinflußt werden, sondern auf große Verrucae vulgares. Bei einer Patientin, die wegen Lues mit zwei Injektionen Salvarsan ... insgesamt 1,25 - behandelt wurde und als Nebenbefund zahlreiche große, harte Warzen an beiden Handrücken und Vorderarmen aufwies, trockneten diese spontan nach der zweiten Injektion ein, bröckelten allmählich ab und verschwanden vollkommen. Eine Wirkung auf spitze Kon. d ylome der Genitalien war bei dieser Patientin wie in mehreren anderen Fällen nicht zu bemerken. Keine deutliche Wirkung sahen wir ferner bei Psoriasis ${ }^{5}$ ), einem chronischen Pemphigus und einigen Fällen von Lupus vulgaris. Bei letzteren war eine bedeutende Zunahme des Körpergewichts zu verzeichnen. Von zwei Patienten mit Lichen ruber planus war bei einem bisher nur eine geringe objektive Wirkung sichtbar, das Jucken verschwand allerdings in etwa einer Woche fast vollkommen; bei dem anderen nahmen die Herde an Rötung, Sukkulen» bald $a b$ und sollen jetzt, nach etwa sechs Wochen, fast vollkominen geschwundell sein (Mitteilung des behandelnden Arztes). Bei einem stark zerfallenen Karzinom des Gesichtes war das Resultat absolut negativ, ebenso bei verrukösen Wucherungen und Karzinombildungen auf der Basis von Röntgenschädigungen. Zahlreiche multiple Mela nome schienen sich nach zwei Injektionen zum Teil zu bessern. Eine mit 0,3 des Präparates behandelte Leukämie zeigte keine Veränderung des Blutibilles.

Vergleichen wir die oben ausführlich wiedergegebenen Resultate der Bellandlung syphilitischer Erscheinungen durch Salvarsan mit den Erfolgen, die mit unseren bisher gebrauchten wirksamsten Mitteln, denl Quecksilber und Jod, erreicht werden, so läßt sich nicht verkennen, daß das neue Mittel in der Abheilung der Krankheits erscheinungen in allen Stadien der Syphilis den wirksamsten Präparaten der Quecksilberbehandlung zum mindesten ebenbürtig erscheint, in der Abheilung der Haut- und namentlich der Schleimhauterscheinungen, ferner in der Beeinflussung der auf Lues beruhenden Kopf- und Knochenschmerzen ihnen überlegen ist. Selbstverständlich müssen wir bei der Kürze der bisherigen Beobachtungszeit mit unserem Urteil über die Nachhaltigkeit der Wirkung noch zurïckhalten. Wir möchten jodoch, namentlich auch unter Berücksichtigung der Beeinflussung der Wassermannschen Reaktion bei reiner und konbinierter Behandlung, die Ansicht aussprechen, daß, sclange uns nicht mehrjährige Beobachtungsresultate zur Verfügung stehen, eine Kombination oder ein Alternieren mit Quecksilberbehandlung in allgemeinen ratsam erscheinen dürfte. Diese kombinierte Behandlung ( $\mathrm{Hg}$ und Salvarsan) ist von Hoffmann zuerst (bereits in seinem Vortrag am 18. Juli 1910) empfohlen worden, und zwar besonders zux frühzeitigen Abortivbehandlung der Syphilis, wo durch die Anwendung der beiden wirksamen Faktoren eine erhöhte Aussicht gegeben ist, mit einen Schlage die Lues zu kupieren. Läßt sich gegenwärtig über den Erfolg einer derartigen Behandlung auch noch nichts Bestimmtes sagen, so ist die große Reihe derart behandelter Fälle, die bisher frei

1) Darnnter 1 vollstăndiger Versager - ${ }^{2}$ ) Beide 5 Monate beobachtet, einer bekam 2 Injektionen (zusaminen $1,2 \mathrm{~g}$ ). $-{ }^{3}$ ) 1 wird nur voräbergehend schwach +. Davon nur 2 klinische Rezidive.

5) Neuerdings wurde in zwei Fällen von Psoriasis, die anderwärts zwei Injektionen von Salvarsan erhalten hatten, ein akuter Ausbruch der Schuppenfechte mit Neigung zu exfoliierender Dermatitis beobachtet. 
von Erscheinungen und nach der Wassermannschen Reaktion negativ geblieben sind, doch bemerkenswert. Ferner scheint uns die kombinierte Behandlung angezeigt bei hartnäckigeren Fällen der anderen Stadien ${ }^{1}$ ) und bei tertiärer Syphilis, wenn Salvarsan allein die Wassermannsche Reaktion nicht in die negative Phase überzuführen vermag. In solchen Fällen, wo eine intensivere Anwendung des Quecksilbers am Platze, aber wegen bestehender Idiosynkrasie oder mit Rücksicht auf den Allgemeinzustand nicht durchführbar ist, wird das Salvarsan einen vollkommenen Ersatz bilden. Auffallend war uns die auf die Injektion oft folgende, manchmal enorme Gewichtszunahme, bis zu $15 \mathrm{~kg}$ in drei Wochen, die bei sehr vielen unserer mit Salvarsan behandelten Patienten konstatiert werden konnte.

Eine Eigenschaft des Salvarsans verdient aber noch ganz besonders hervorgehoben zu werden, weil sie keines der bisher bekannten Antisyphilitica besitzt; sie ist uns so wichtig erschienen, daß wir sie auch im Tierexperiment geprüft haben. Es ist die schon oben erwähnte ungemein rasche Einwirkung auf die offenen und infektiösen Produkte der rezenten Syphilis und die Vernichtung der Spirochäten i m Sekret derselhen, eine Wirkung, die das Salvarsan als eine besonders wirksame Waffe zur Bekämpfung der Ausbreitung der Syphilis erscheinen läßt. Denn die Quecksilberpräparate besitzen ebensowenig wie das Atoxyl diese Eigenschaft in dem Maße, wie durch mikroskopische Untersuchung und Experimente mehrfach - auch von Hoff mann - nachgewiesen worden ist. Und noch nach einer anderen Richtung hin scheint die experimentelle Prüfung des neuen Mittels dessen hohe Bewertung darzutun gegenüber den bisherigen Antisyphilitica, nämlich in seiner präventiven Wirkung. Dies war, wie Hoffmann schon in seiner ersten Mitteilung betont hat, von vornherein zu erwarten, da das Atoxyl sich nach Tierversuchen von Uhlenhuth und Hoffmann hierfïr besonders bewährt hatte. Vorläufig liegt nur ein vollendeter und verwertbarer Versuch vor. Von einer Reihe von zehn in die vordere Augenkammer geimpften Kaninchen erkrankten acht unbehandelte nach rund vier Wochen an spezifischer Keratitis; zwei, die 24 Stunden nach der Impfung mit 0,02 Salvarsan pro $\mathrm{kg}$ intramuskulär behandelt worden waren, sind jetzt bereits vier Monate vollkommen frei von allen Krankheitserscheinungen geblieben.

Schlußsätze. 1. Dem Salvarsan kommt schon bei einmaliger, in höherem Grade bei zweimaliger Injektion in allen Stadien der Syphilis (abgesehen von den sog. metasyphilitischen Erkrankungen) eine auffallende, den Einfluß des Quecksilbers erreichende oder gar übertreffende Wirkung zu.

2. Die besten Applikationsarten sind nach unserer Erfahrung die intraglutäale Injektion einer schwach alkalischen, leicht trüben Lösung nach Alt und die intravenöse Injektion nach Weintraud.

3. Daß durch das Salvarsan schon bei einer Injektion eine Therapia sterilisans magna zustande kommt, ist bisher nicht bewiesen. Das langsame Schwinden der Wassermannschen Reaktion und die schon jetzt häufig beobachteten Rezidive zeigen, da $B$ dies in einer Reihe von Fällen jedenfalls nicht gelungen ist.

4. Gefährliche Nebenwirkungen, wie dauernde Schädigungen von Organen, scheinen unseren Erfahrungen nach dem Mittel bei richtiger Dosierung nicht zuzukommen; ob frühzeitige Nervenerkrankungen (Neuritis optica, Acusticuserkrankung, Fazialislähmung etc.) häufiger und früher auftreten als sonst, läßt sich gegenwärtig noch nicht entscheiden. Spät auftretende Arzneiexantheme kommen ebenso wie Zoster nach subkutaner und intramuskulärer, aber auch nach intravenöser Injektion vor.

5. Die Wassermannsche Reaktion wird am schnellsten und nach unseren Beobachtungen stets - bei primäres Lues umgestimmt, langsamer und nicht immer bei sekundär syphili-

1) Bei der Lues maligna erreichen wir nach unserer Erfahrung durch Kalomelinjektionen mit oder ohne begleitende Jodmedikation in kurzer Zeit fast durchweg schnelle Abheilung der sonst hartnäckigen Haut- und Schleimhauterscheinungen und meist in 6--8 Wochen negative Wassermannsche Reaktion. Vorziiglich wirkt in schweren Fällen Salvarsan (intravenös) + Kalomelinjektionen. tischen Erscheinungen und Lues maligna, noch zögernder bei tertiärer und tardiver kongenitaler Lues, wo die Umwandlung in unseren Fällen bisher nicht erreicht wurde.

6. Besondere Vorteile der Salvarsanbehandlung sind:

a) der schnelle Nachlaß der Sekretion und die rasche Ueberhäutung der infektiösen syphilitischen Effloreszenzen und

b) das schnelle Verschwinden der Spirochäten, das von uns nicht nur im Oberflächensekret, sondern auch im Gewebssaft aus der Tiefe und durch Ueberimpfung im Tierexperiment festgestellt werden konnte.

Diese Eigenschaften gewähren uns schon jetzt die Möglichkeit, die Kranken schneller aus dem Krankenhaus zu entlassen, und dürften voraussichtlich für die systematische Bekämpfung und Eindämmung der Syphilis Bedeutung gewinnen.

7. Gleichzeitige Anwendung des Salvarsans und Quecksilbers ist, wie Hoff mann zuerst gezeigt hat, gut durchführbar; nach seinem Vorschlag empfiehlt sie sich vor allem zur Abortivbehandlung der frischen syphilitischen Infektion und auch in den Fällen von Spätlues, in denen Salvarsan allein die Umwandlung der Wassermannschen Reaktion nicht herbeizuführen vermag. 